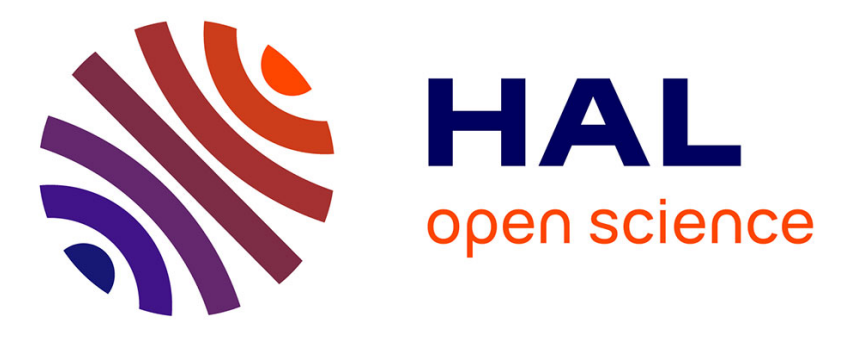

\title{
Sotatercept for the Treatment of Pulmonary Arterial Hypertension
}

\author{
Marc Humbert, Vallerie Mclaughlin, J. Simon R. Gibbs, Mardi \\ Gomberg-Maitland, Marius Hoeper, Ioana Preston, Rogerio Souza, Aaron \\ Waxman, Pilar Escribano Subias, Jeremy Feldman, et al.
}

\section{To cite this version:}

Marc Humbert, Vallerie Mclaughlin, J. Simon R. Gibbs, Mardi Gomberg-Maitland, Marius Hoeper, et al.. Sotatercept for the Treatment of Pulmonary Arterial Hypertension. New England Journal of Medicine, 2021, 384 (13), pp.1204-1215. 10.1056/NEJMoa2024277 . hal-03270003

\section{HAL Id: hal-03270003 https://hal.science/hal-03270003}

Submitted on 24 Jun 2021

HAL is a multi-disciplinary open access archive for the deposit and dissemination of scientific research documents, whether they are published or not. The documents may come from teaching and research institutions in France or abroad, or from public or private research centers.
L'archive ouverte pluridisciplinaire HAL, est destinée au dépôt et à la diffusion de documents scientifiques de niveau recherche, publiés ou non, émanant des établissements d'enseignement et de recherche français ou étrangers, des laboratoires publics ou privés. 
PULSAR Trial Investigators: David Badesch, Marc Humbert, Vallerie McLaughlin, Simon Gibbs, Mardi Gomberg-, Maitland Maitland, Marius Hoeper, Ioana Preston, Rogerio de Souza, Aaron Waxman, Yochai Adir, Marina Andrade Lima, Rahul Argula, David Baratz, Joan Barberá, Amir Bar-Shai, Cristina Berasagui, Laurent Bertoletti, Daniela Blanco, Arnaud Bourdin, Frederico Campos, Colin Church, Jose Manuel Cifrian Martinez, John Gerry Coghlan, Teresa Demarco, Stephan Eisenmann, Peter Engel, Pilar Escribano Subias, John Feenstra, Jeremy Feldman, Michael Halank, Luke Howard, Anne Keogh, Mordechai Kramer, Tobias Lange, Gisela Meyer, David Montani, Karen Olsson, Jaquelina Ota Arakaki, Christophe Pison, Glenn Reeves, Franz Rischard, Zeenat Safdar, Rajeev Saggar, Michael Segel, Javier Segovia Cubero, Leslie Spikes, David Shitrit, John Wheatley, Hubert Wirtz, Bruce Brundage, Ian Ford, Ellis Neufeld, Michael McGoon

\title{
Sotatercept for the Treatment of Pulmonary Arterial Hypertension
}

\author{
Marc Humbert, M.D., Ph.D., Vallerie McLaughlin, M.D., J. Simon R. Gibbs, M.D., \\ Mardi Gomberg-Maitland, M.D., Marius M. Hoeper, M.D., loana R. Preston, M.D., \\ Rogerio Souza, M.D., Ph.D., Aaron Waxman, M.D., Ph.D., \\ Pilar Escribano Subias, M.D., Ph.D., Jeremy Feldman, M.D., Gisela Meyer, M.D., \\ David Montani, M.D., Ph.D., Karen M. Olsson, M.D., Solaiappan Manimaran, Ph.D., \\ Jennifer Barnes, Ph.D., Peter G. Linde, M.D., Janethe de Oliveira Pena, M.D., Ph.D., \\ and David B. Badesch, M.D., for the PULSAR Trial Investigators*
}

ABSTRACT

BACKGROUND

Pulmonary arterial hypertension is characterized by pulmonary vascular remodeling, cellular proliferation, and poor long-term outcomes. Dysfunctional bone morphogenetic protein pathway signaling is associated with both hereditary and idiopathic subtypes. Sotatercept, a novel fusion protein, binds activins and growth differentiation factors in the attempt to restore balance between growth-promoting and growth-inhibiting signaling pathways.

METHODS

In this 24-week multicenter trial, we randomly assigned 106 adults who were receiving background therapy for pulmonary arterial hypertension to receive subcutaneous sotatercept at a dose of $0.3 \mathrm{mg}$ per kilogram of body weight every 3 weeks or $0.7 \mathrm{mg}$ per kilogram every 3 weeks or placebo. The primary end point was the change from baseline to week 24 in pulmonary vascular resistance.

RESULTS

Baseline characteristics were similar among the three groups. The least-squares mean difference between the sotatercept $0.3-\mathrm{mg}$ group and the placebo group in the change from baseline to week 24 in pulmonary vascular resistance was $-145.8 \mathrm{dyn} \cdot \mathrm{sec} \cdot \mathrm{cm}^{-5}$ (95\% confidence interval [CI], -241.0 to $-50.6 ; \mathrm{P}=0.003$ ). The least-squares mean difference between the sotatercept $0.7-\mathrm{mg}$ group and the placebo group was -239.5 dyn $\cdot \mathrm{sec} \cdot \mathrm{cm}^{-5}$ (95\% CI, -329.3 to $\left.-149.7 ; \mathrm{P}<0.001\right)$. At 24 weeks, the least-squares mean difference between the sotatercept 0.3 -mg group and the placebo group in the change from baseline in 6-minute walk distance was $29.4 \mathrm{~m}$ (95\% CI, 3.8 to 55.0). The least-squares mean difference between the sotatercept $0.7-\mathrm{mg}$ group and the placebo group was $21.4 \mathrm{~m}$ (95\% CI, -2.8 to 45.7$)$. Sotatercept was also associated with a decrease in N-terminal pro-B-type natriuretic peptide levels. Thrombocytopenia and an increased hemoglobin level were the most common hematologic adverse events. One patient in the sotatercept $0.7-\mathrm{mg}$ group died from cardiac arrest.

CONCLUSIONS

Treatment with sotatercept resulted in a reduction in pulmonary vascular resistance in patients receiving background therapy for pulmonary arterial hypertension. (Funded by Acceleron Pharma; PULSAR ClinicalTrials.gov number, NCT03496207.) 


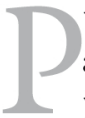
ULMONARY ARTERIAL HYPERTENSION IS a rare, progressive disorder characterized by pulmonary vascular remodeling, resulting in high pulmonary artery pressure and progressive right ventricular dysfunction..$^{1-3}$ Current treatments, which target the prostacyclin, endothelin-1, or nitric oxide pathways, slow disease progression. However, the 5-year survival rate of approximately $60 \%$ highlights the need for therapies targeting alternative pulmonary vascular remodeling pathways. ${ }^{1,4-7}$

Mutations in bone morphogenetic protein (BMP) receptor type 2 (BMPR2) - a member of the transforming growth factor $\beta$ (TGF- $\beta$ ) superfamily - are a major factor underlying heritable pulmonary arterial hypertension; the BMPR-II signaling pathway is also important in development of the disease in patients without heritable disease. ${ }^{8-11}$ Owing to the role of the BMPR-II pathway in maintaining endothelial integrity in pulmonary arteries, mutations that reduce signaling in this pathway promote endothelial dysfunction, increased cellular proliferation, and pulmonary vascular remodeling.,10,12-16

Sotatercept is a novel, first-in-class fusion protein composed of the extracellular domain of the human activin receptor type IIA fused to the Fc domain of human IgG1. Sotatercept acts as a ligand trap for members of the TGF- $\beta$ superfamily, thus restoring balance between the growth-promoting activin growth differentiation factor pathway and the growth-inhibiting BMP pathway (Fig. 1). ${ }^{17}$ Sotatercept has been evaluated in healthy volunteers, in patients with hematologic disorders, and in patients with conditions characterized by a dysfunctional TGF- $\beta$ superfamily signaling pathway, including bone loss, chemotherapy-induced anemia, multiple myeloma, myelodysplastic syndromes, $\beta$-thalassemia, and end-stage kidney disease. ${ }^{18-24}$ In the PULSAR trial, we investigated the safety and efficacy of sotatercept in patients with pulmonary arterial hypertension who were receiving background therapy for pulmonary hypertension.

\section{METHODS}

\section{TRIAL DESIGN AND OVERSIGHT}

PULSAR is a multicenter, randomized, doubleblind, phase 2 trial that includes a 24-week placebo-controlled treatment period, followed by an 18-month active-drug extension period that is currently ongoing. Here, we report the main results from the 24 -week placebo-controlled period.

A steering committee designed the trial in collaboration with the sponsor, Acceleron Pharma. An institutional review board or independent ethics committee at each trial center approved the protocol. An independent data and safety monitoring committee reviewed unblinded safety data collected 6 weeks after the first dose of sotatercept or placebo was administered. The trial was conducted in accordance with the principles of the Declaration of Helsinki, the international ethical guidelines of the Council for International Organizations of Medical Sciences, the Good Clinical Practice guidelines of the International Council for Harmonisation, and applicable laws and regulations. ${ }^{25-27}$

In addition to its role in the design of the trial, the sponsor selected the participating trial centers; oversaw the conduct and monitoring of the trial, which were performed by a contract research organization (PPD); received and maintained the trial database; and performed all the data analyses. No independent analyses of the data were performed. The academic authors had the authority to request additional analyses. All the authors vouch for the completeness and accuracy of the data and analyses and for the fidelity of the trial to the protocol and the statistical analysis plan, which are available with the full text of this article at NEJM.org.

\section{PATIENTS}

Eligible patients had confirmed pulmonary arterial hypertension (group 1 of the updated World Health Organization [WHO] classification of pulmonary hypertension) in WHO functional class II or III (classes range from I to IV, with higher numbers indicating greater functional limitations), excluding the subtypes associated with portopulmonary disease, schistosomiasis, and human immunodeficiency virus infection. ${ }^{2}$ A full list of inclusion and exclusion criteria is provided in the Supplementary Appendix, available at NEJM.org. Patients were receiving stable background therapy for pulmonary arterial hypertension for at least 90 days before enrollment in the trial and continued to receive the treatment during the course of the trial. The treatment consisted of monotherapy, double therapy, or triple therapy with combinations of endothelin- 


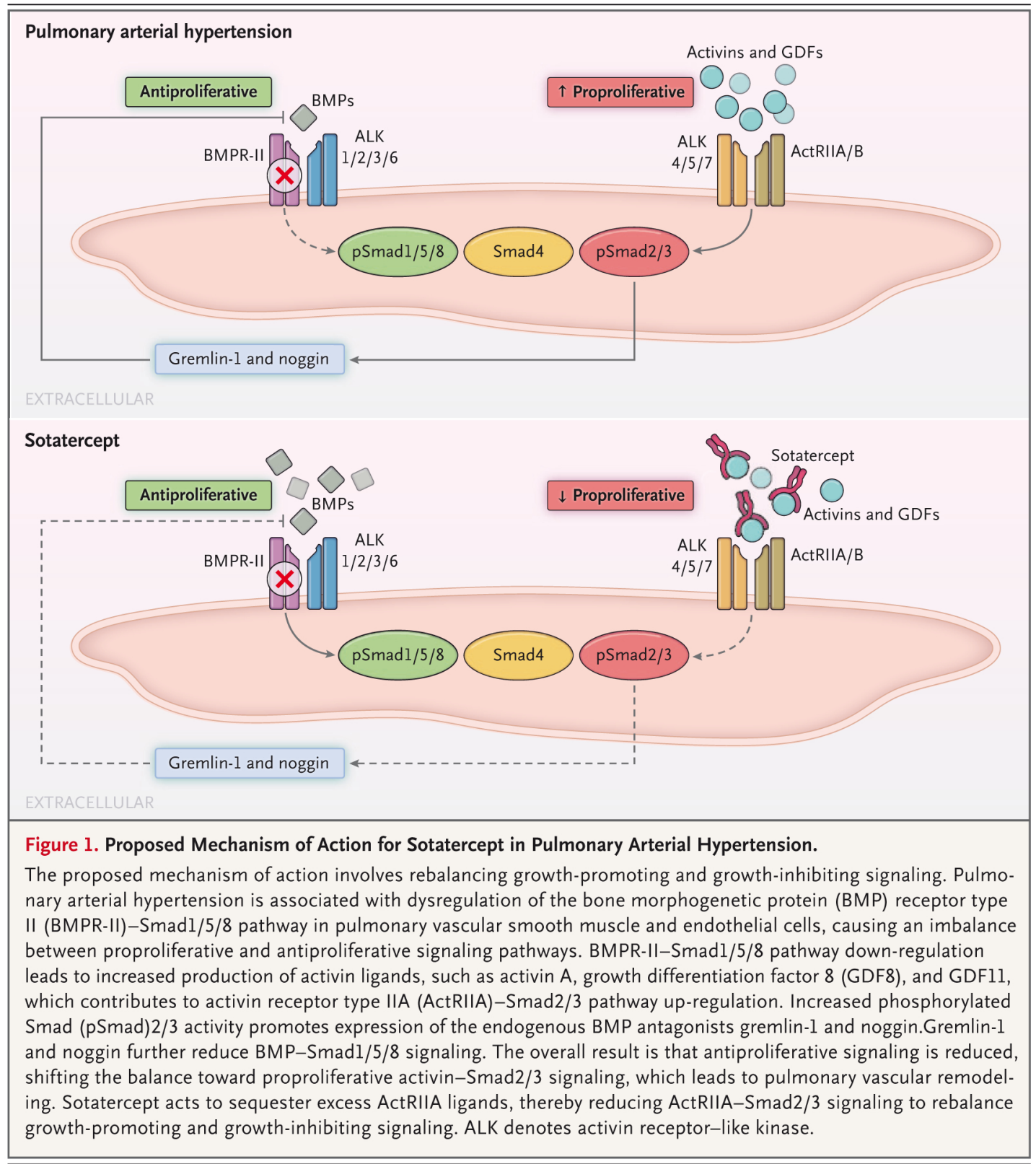

receptor antagonists, phosphodiesterase-5 inhibitors, soluble guanylate cyclase stimulators, prostacyclin analogues, or prostacyclin-receptor agonists. All the patients provided written informed consent.

\section{PROCEDURES}

Initially, eligible patients were stratified according to baseline WHO functional class and were randomly assigned in a 1:1:1 ratio by an interactive response technology system to one of three groups: placebo, sotatercept at a dose of $0.3 \mathrm{mg}$ per kilogram of body weight, or sotatercept at a

dose of $0.7 \mathrm{mg}$ per kilogram. During the trial, the randomization ratio was changed to $3: 3: 4$ to increase the statistical power with respect to the sotatercept $0.7-\mathrm{mg}$ group; 7 patients had been enrolled at the time of this change. Sotatercept or placebo (saline) was administered by means of subcutaneous injection every 21 days.

Safety and efficacy were assessed at screening and every 3 weeks thereafter for 24 weeks. Adverse events were recorded from the time of screening until 8 weeks after the end of the placebo-controlled treatment period. Patients 
who completed the placebo-controlled treatment period continued directly into the 18-month extension period; the recording of adverse events continues in the extension period. Dose modifications were planned in accordance with guidance from the European Medicines Agency (as outlined in Table S1 in the Supplementary Appendix) for any occurrences of leukopenia, neutropenia, or thrombocytopenia or increases in hemoglobin levels or blood pressure. These adverse events have been reported in previous trials of sotatercept. Patients who discontinued sotatercept or placebo or withdrew consent were asked to return for the end-of-trial visit.

\section{END POINTS}

The primary end point was the change from baseline to week 24 in pulmonary vascular resistance. The key secondary end point was the change from baseline to week 24 in 6-minute walk distance. Other secondary efficacy end points included the change from baseline to week 24 in $\mathrm{N}$-terminal pro-B-type natriuretic peptide (NT-proBNP) level, systolic excursion of the tricuspid annular plane (assessed by echocardiography), WHO functional class, clinical worsening, scores on the Cambridge Pulmonary Hypertension Outcome Review, and scores on the 36-Item Short-Form Health Survey (SF-36). Safety end points included adverse events and clinical laboratory test results. A complete list of the trial end points is provided in Table S2.

Pulmonary vascular resistance was calculated with the use of the mean pulmonary artery pressure, the pulmonary artery wedge pressure, and the cardiac output, all of which were measured by catheterization of the right side of the heart at screening and at week 24 of the placebo-controlled treatment period. Echocardiograms (two-dimensional) were obtained according to a prespecified protocol and were approved, reviewed, and analyzed in a blinded manner at a central laboratory (BioTel Research). End points were assessed at baseline and at selected trial visits. Adverse events were graded according to the National Cancer Institute Common Terminology Criteria for Adverse Events (CTCAE), version 4.0. The methods used for catheterization and echocardiography, as well as descriptions of the end point of clinical worsening and the grades used in the CTCAE, are provided in the Supplementary Appendix.

\section{STATISTICAL ANALYSIS}

The sample-size calculation for the trial was based on the following assumptions: a baseline mean $( \pm S D)$ pulmonary vascular resistance of $800 \pm 400 \mathrm{dyn} \cdot \mathrm{sec} \cdot \mathrm{cm}^{-5}$; a $30 \%$ reduction in mean pulmonary vascular resistance $\left(240 \mathrm{dyn} \cdot \mathrm{sec} \cdot \mathrm{cm}^{-5}\right)$ at 24 weeks in the sotatercept groups and no change in the placebo group; a one-sided alpha level of 0.10 and $80 \%$ power to detect the difference among groups; and dropout rates of $15 \%$ in the placebo group, $15 \%$ in the sotatercept $0.3-\mathrm{mg}$ group, and $35 \%$ in the sotatercept $0.7-\mathrm{mg}$ group. The estimated dropout rate in the sotatercept 0.7-mg group was higher owing to potential hemoglobin increases, as determined by pharmacokinetic modeling. On the basis of these assumptions, we estimated a sample size of approximately 100 patients to be randomly assigned in the ratio of 3:3:4 across the three groups.

All the efficacy and safety analyses were performed in the intention-to-treat population, which included all the patients who underwent randomization. The primary end point was analyzed with the use of an analysis of covariance (ANCOVA) model, with the randomization factor and baseline value as covariates. The analysis was performed with a closed testing procedure for correction of multiplicity and a hierarchical testing procedure. First, the sotatercept $0.7-\mathrm{mg}$ group was compared with the placebo group, followed by a comparison of the sotatercept $0.3-\mathrm{mg}$ group with the placebo group. Multiple imputation was used to handle missing data for all the primary and secondary efficacy end points that were assessed as continuous variables. Other end points were summarized with the use of descriptive statistics and analyzed with an ANCOVA model, as appropriate. Conventional two-sided $\mathrm{P}$ values are presented for the primary end point; no $\mathrm{P}$ values are presented for secondary and other end points. Sensitivity analyses of the primary and key secondary end points were performed; in one analysis, only the patients who were receiving double or triple therapy for pulmonary arterial hypertension at baseline were included, and in an additional analysis, missing data were imputed with the use of the last-observation-carried-forward method. The underlying normality assumption for ANCOVA was tested with the use of the ShapiroWilk test. If the assumption of normality was rejected, the Hodges-Lehmann analysis was used to present the location shift value. Results are 


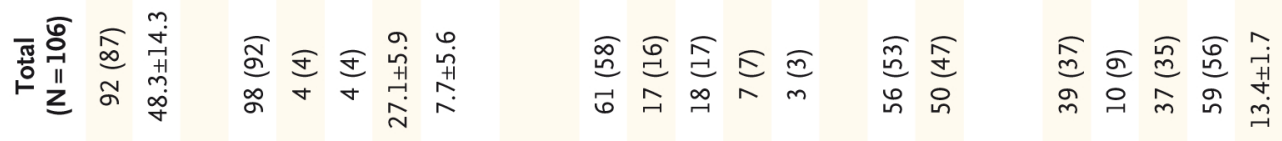

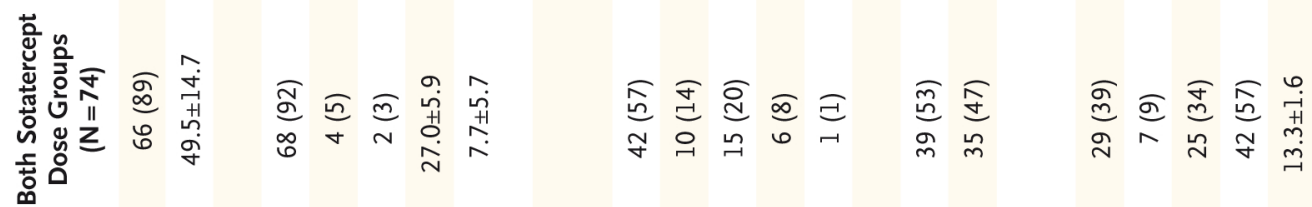

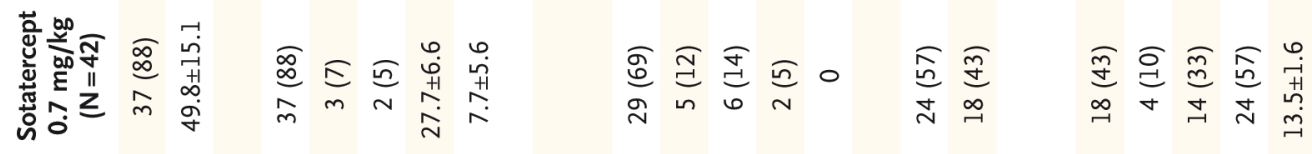

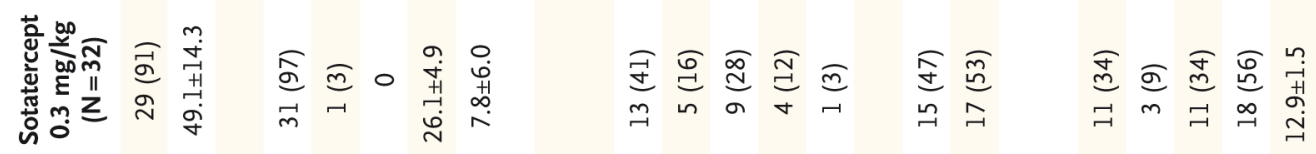

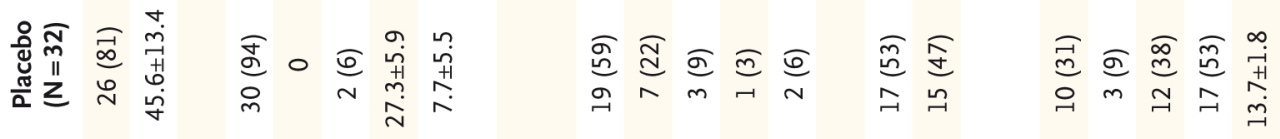

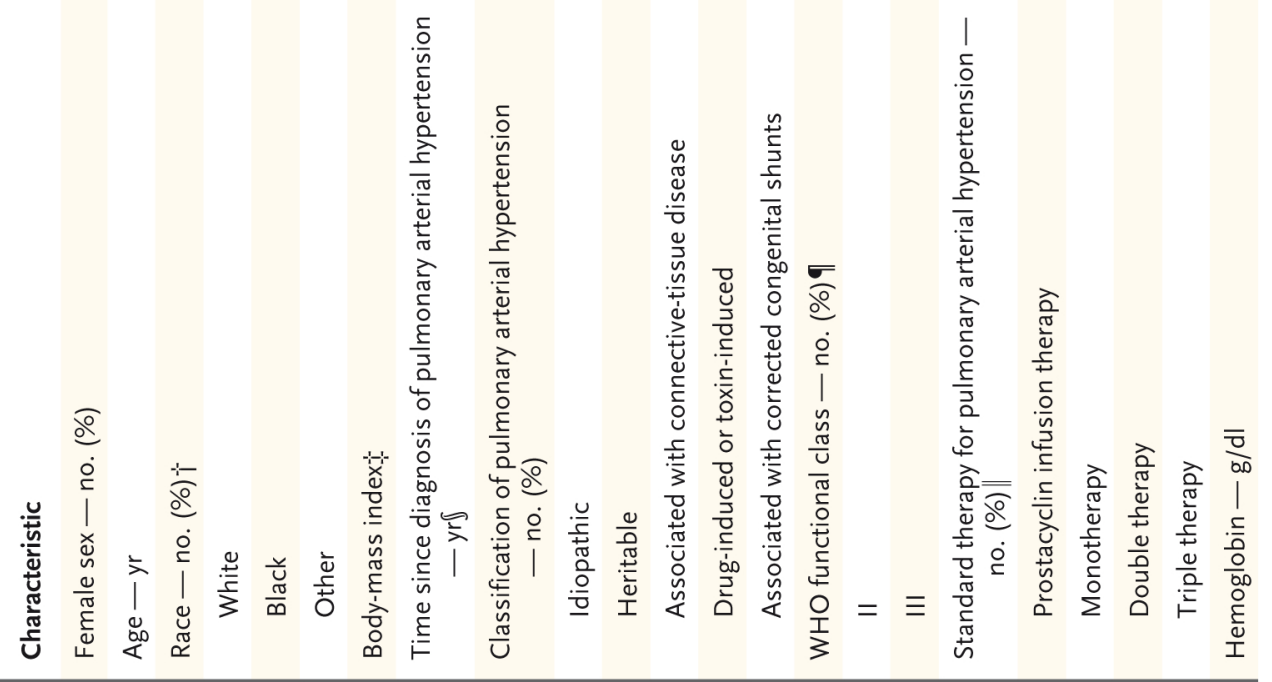




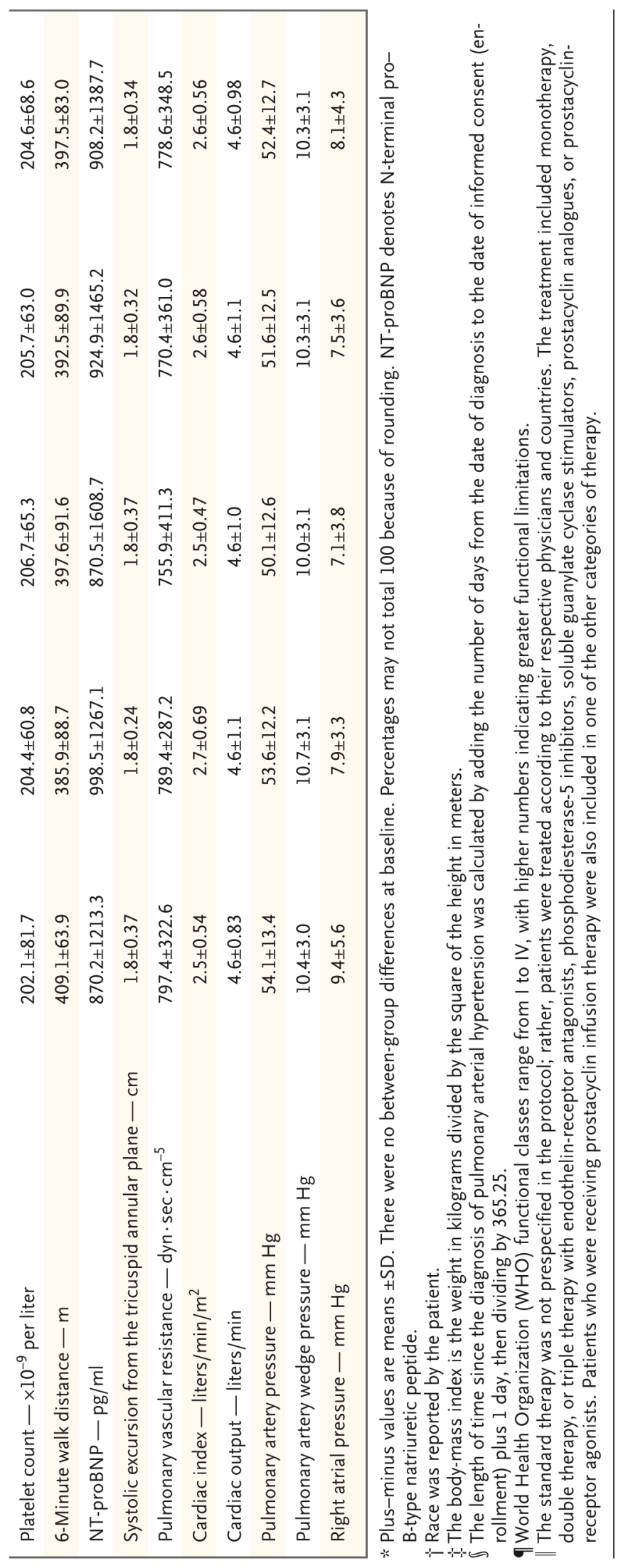


Placebo Sotatercept $0.3 \mathrm{mg} / \mathrm{kg}$ Sotatercept $0.7 \mathrm{mg} / \mathrm{kg}$

A Change from Baseline to Week 24 in Pulmonary Vascular Resistance

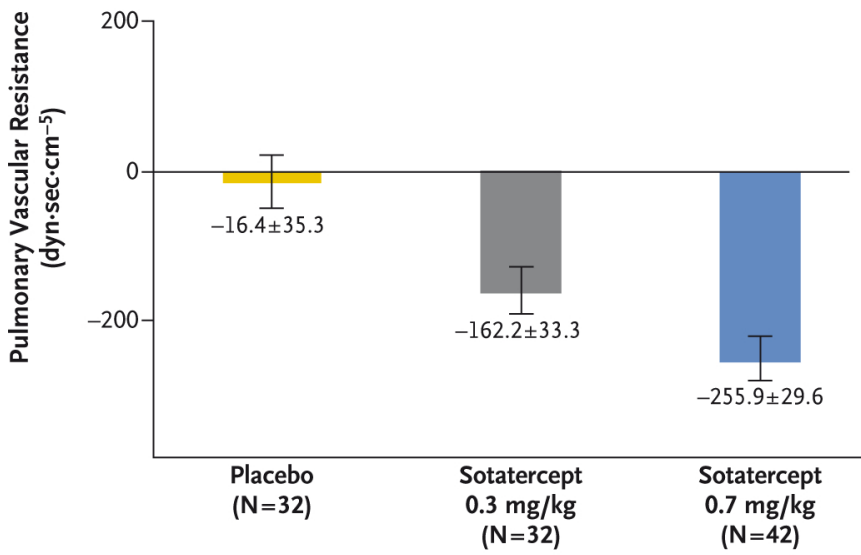

B Pulmonary Vascular Resistance at Baseline and at Week 24 of the Placebo-Controlled Period

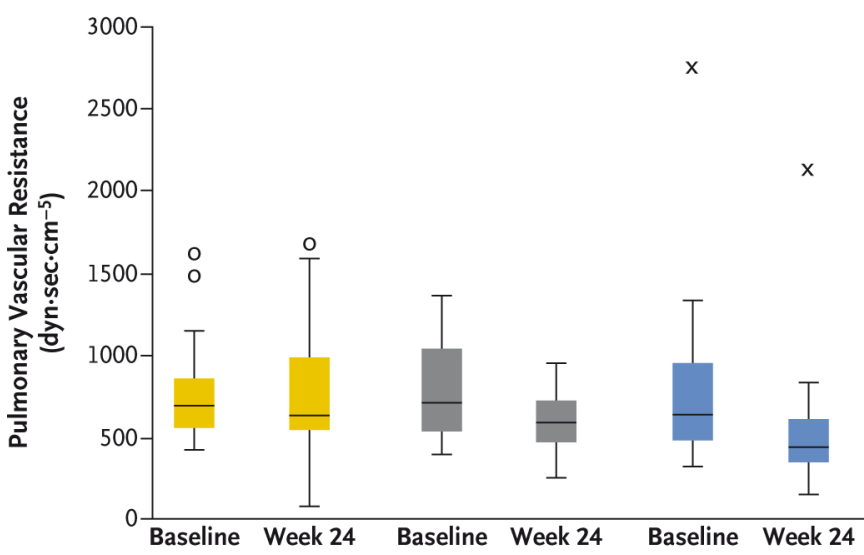

Figure 2. Change in Pulmonary Vascular Resistance from Baseline to Week 24 in the Intention-to-Treat Population.

Panel A shows the least-squares mean change from baseline to week 24 in pulmonary vascular resistance in each group. The least-squares mean difference between the sotatercept $0.3-\mathrm{mg}$ group and the placebo group was $-145.8 \mathrm{dyn} \cdot \mathrm{sec} \cdot \mathrm{cm}^{-5}(95 \% \mathrm{Cl},-241.0$ to -50.6$)$. The least-squares mean difference between the sotatercept $0.7-\mathrm{mg}$ group and the placebo group was $-239.5 \mathrm{dyn} \cdot \mathrm{sec} \cdot \mathrm{cm}^{-5}(95 \% \mathrm{Cl},-329.3$ to -149.7$)$; the $0.7-\mathrm{mg}$ group had a $34 \%$ reduction from baseline. I bars indicate standard errors. Panel $\mathrm{B}$ shows pulmonary vascular resistance at baseline and at the end of the placebocontrolled treatment period (week 24) in the three groups. The horizontal line in the boxes represents the median, and the bottom and top of the boxes represent the first and third quartiles, respectively. I bars indicate the minimum and maximum range, excluding outliers. Outliers are represented by single point markers (o and $x$ ). reported as point estimates and $95 \%$ confidence intervals. The widths of the confidence intervals have not been adjusted for multiple comparisons, so the intervals should not be used to infer definitive treatment effects for the secondary outcomes or subgroup analyses.

\section{R E S U LT S}

\section{BASELINE CHARACTERISTICS AND FOLLOW-UP}

From June 2018 through July 2019, a total of 163 patients were screened, of whom 106 underwent randomization at 43 centers in eight countries. Patients were randomly assigned to receive placebo (32 patients), sotatercept at a dose of $0.3 \mathrm{mg}$ per kilogram (32 patients), or sotatercept at a dose of $0.7 \mathrm{mg}$ per kilogram (42 patients), in addition to stable background therapy for pulmonary arterial hypertension (Fig. S1). The reasons for screening failure for the 57 patients who were excluded are provided in Table S3. Demographic and baseline clinical characteristics were similar among the groups, showing a relatively young patient population (mean $[ \pm S D]$ age, $48.3 \pm 14.3$ years) with moderate-to-severe pulmonary arterial hypertension. In total, 59 of the 106 patients $(56 \%)$ were receiving triple therapy and $39(37 \%)$ were receiving prostacyclin infusion therapy (Table 1).

The last patient completed the 24-week placebocontrolled treatment period on December 17, 2019, and data were unblinded for analysis on January 8, 2020. Data on cumulative drug exposure during the placebo-controlled treatment period are provided in Table S4.

\section{PRIMARY END POINT}

In the intention-to-treat population at week 24 , the least-squares mean change from baseline in pulmonary vascular resistance was a decrease of $162.2 \mathrm{dyn} \cdot \mathrm{sec} \cdot \mathrm{cm}^{-5}$ in the sotatercept $0.3-\mathrm{mg}$ group and a decrease of $255.9 \mathrm{dyn} \cdot \mathrm{sec} \cdot \mathrm{cm}^{-5}$ in the sotatercept $0.7-\mathrm{mg}$ group, as compared with a decrease of $16.4 \mathrm{dyn} \cdot \mathrm{sec} \cdot \mathrm{cm}^{-5}$ in the placebo group. The least-squares mean difference between the sotatercept $0.3-\mathrm{mg}$ group and the placebo group at week 24 was $-145.8 \mathrm{dyn} \cdot \mathrm{sec} \cdot \mathrm{cm}^{-5}$ (95\% confidence interval [CI], -241.0 to -50.6 ; $\mathrm{P}=0.003$ ), and the least-squares mean difference between the sotatercept $0.7-\mathrm{mg}$ group and the placebo group was $-239.5 \mathrm{dyn} \cdot \mathrm{sec} \cdot \mathrm{cm}^{-5}(95 \% \mathrm{CI}$, -329.3 to -149.7 ; P<0.001) (Fig. 2 and Table 2). Sensitivity analyses showed similar results (Table S5). This treatment effect was consistent across a range of prespecified subgroups, including those that were defined according to 


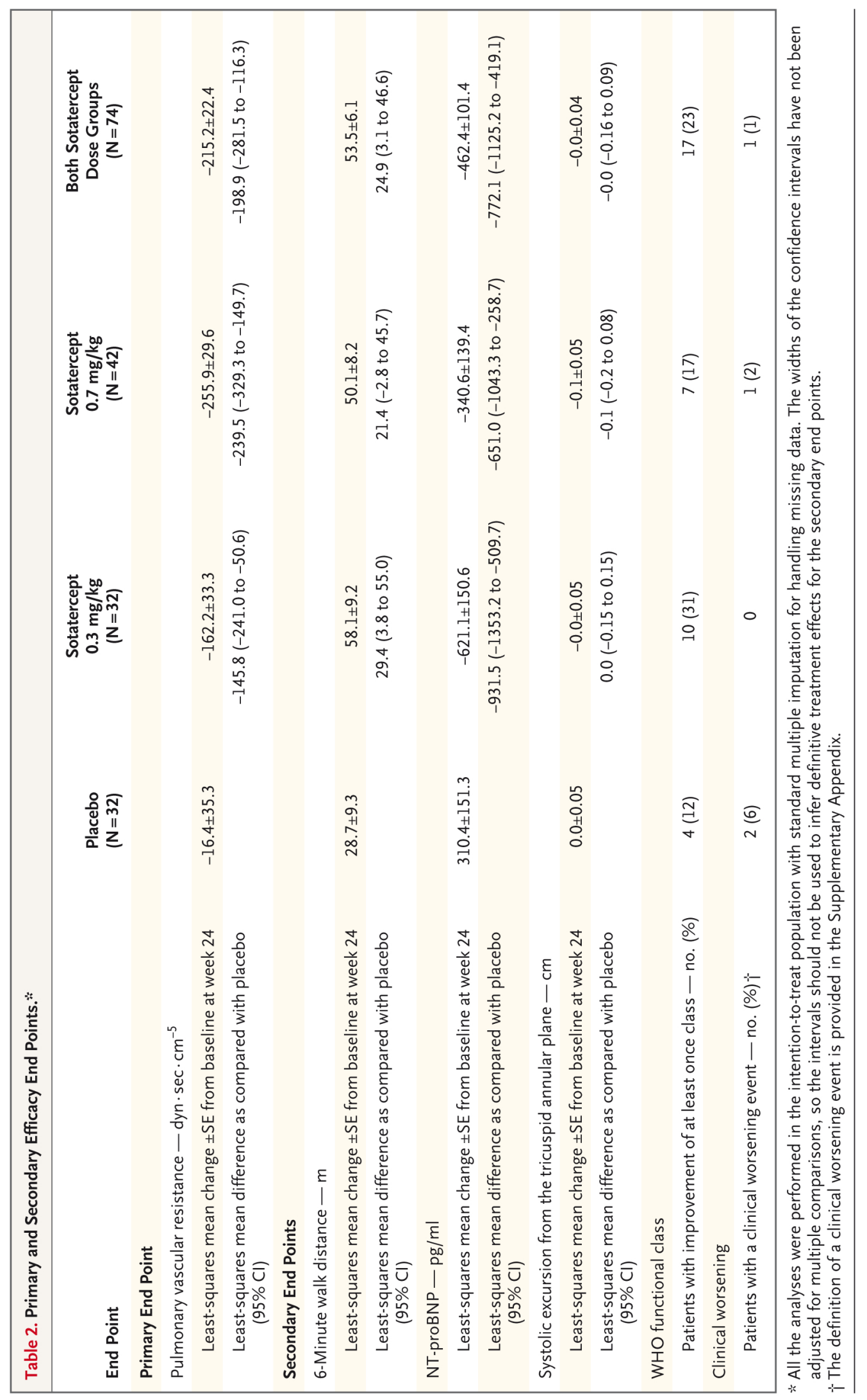




\begin{tabular}{|c|c|c|c|}
\hline Variable & $\begin{array}{l}\text { Placebo } \\
(\mathrm{N}=32)\end{array}$ & $\begin{array}{c}\text { Sotatercept } \\
0.3 \mathrm{mg} / \mathrm{kg} \\
(\mathrm{N}=32)\end{array}$ & $\begin{array}{c}\text { Sotatercept } \\
0.7 \mathrm{mg} / \mathrm{kg} \\
(\mathrm{N}=42)\end{array}$ \\
\hline Any adverse event — no. (\%) & $28(88)$ & $29(91)$ & $34(81)$ \\
\hline \multicolumn{4}{|l|}{$\begin{array}{l}\text { Adverse event occurring in } \geq 10 \% \text { of patients in any } \\
\text { group - no. (\%) }\end{array}$} \\
\hline Headache & $5(16)$ & $8(25)$ & $6(14)$ \\
\hline Diarrhea & $4(12)$ & $7(22)$ & $6(14)$ \\
\hline Peripheral edema & $5(16)$ & $3(9)$ & $5(12)$ \\
\hline Dizziness & $3(9)$ & $5(16)$ & $4(10)$ \\
\hline Fatigue & $6(19)$ & $2(6)$ & $4(10)$ \\
\hline Hypokalemia & $4(12)$ & $3(9)$ & $5(12)$ \\
\hline Nausea & $4(12)$ & $3(9)$ & $5(12)$ \\
\hline Adverse event of special interest — no. (\%) & 0 & $3(9)$ & $6(14)$ \\
\hline Leukopenia & 0 & $1(3)$ & $1(2)$ \\
\hline Neutropenia & 0 & 0 & $1(2)$ \\
\hline Thrombocytopenia & 0 & $2(6)$ & $5(12)$ \\
\hline Serious adverse event - no. (\%) & $3(9)$ & $2(6)$ & $10(24)$ \\
\hline $\begin{array}{l}\text { Adverse event leading to discontinuation of sotater- } \\
\text { cept or placebo - no. (\%) }\end{array}$ & $1(3)$ & $2(6)$ & $3(7)$ \\
\hline $\begin{array}{l}\text { Adverse event leading to withdrawal from the trial } \\
\qquad- \text { no. (\%) }\end{array}$ & $2(6)$ & $1(3)$ & $3(7)$ \\
\hline Adverse event leading to death — no. (\%) & 0 & 0 & $I(2) \dagger$ \\
\hline $\begin{array}{l}\text { Hemoglobin increase reported as an adverse event } \\
\text { — no. (\%) }\end{array}$ & 0 & $1(3)$ & $7(17)$ \\
\hline \multicolumn{4}{|l|}{$\begin{array}{l}\text { Change in hematologic variables from baseline to } \\
\text { week } 24\end{array}$} \\
\hline No. of patients & 30 & 31 & 36 \\
\hline Hemoglobin $-\mathrm{g} / \mathrm{dl}$ & $0.0 \pm 1.1$ & $1.2 \pm 1.2$ & $1.5 \pm 1.1$ \\
\hline Platelet count $-\times 10^{9}$ per liter & $-6.3 \pm 29.1 \div$ & $12.1 \pm 47.7$ & $-12.1 \pm 49.8$ \\
\hline
\end{tabular}

* Plus-minus values are means \pm SD.

$\dagger$ This patient died from cardiac arrest. Preexisting risk factors included hypertension, type 2 diabetes, chronic obstructive pulmonary disease, hyperlipidemia, atrial fibrillation, congestive heart disease, and ischemic heart disease. Concomitant medications included furosemide, spironolactone, ambrisentan, tadalafil, apixaban, bisoprolol, digoxin, gliclazide, linagliptin, pravastatin, and tiotropium.

$\checkmark$ A total of 29 patients in the placebo group were included in the analysis of platelet count.

background therapy (monotherapy or double therapy vs. triple therapy) and according to prostacyclin infusion therapy (yes vs. no) (Fig. S2). The reduction in pulmonary vascular resistance in the sotatercept groups was driven by a decreased mean pulmonary artery pressure (least-squares mean difference of $-8.3 \mathrm{~mm} \mathrm{Hg}$ [95\% CI, -12.7 to -4.0 ] between the sotatercept $0.3-\mathrm{mg}$ group and the placebo group and of $-13.4 \mathrm{~mm} \mathrm{Hg}$ [95\% CI, -17.5 to -9.3 ] between the sotatercept 0.7-mg group and the placebo group) (Table S6). In contrast, pulmonary artery wedge pressure and cardiac output showed minimal changes across the three groups.

\section{SECONDARY EFFICACY END POINTS}

In the intention-to-treat population, the leastsquares mean change from baseline in 6-minute walk distance at week 24 was an increase of $58.1 \mathrm{~m}$ in the sotatercept $0.3-\mathrm{mg}$ group and an increase of $50.1 \mathrm{~m}$ in the sotatercept $0.7-\mathrm{mg}$ group, as compared with an increase of $28.7 \mathrm{~m}$ in the placebo group (Figs. S3 and S4 and Table 2). The least-squares mean difference between the 
sotatercept 0.3-mg group and the placebo group at week 24 was $29.4 \mathrm{~m}$ (95\% CI, 3.8 to 55.0 ), and the least-squares mean difference between the sotatercept $0.7-\mathrm{mg}$ group and the placebo group was $21.4 \mathrm{~m}$ (95\% CI, -2.8 to 45.7$)$. Sensitivity analyses showed similar results (Table S5).

At week 24, the least-squares mean change from baseline in the level of NT-proBNP was a decrease of $621.1 \pm 150.6 \mathrm{pg}$ per milliliter in the sotatercept $0.3-\mathrm{mg}$ group and a decrease of $340.6 \pm 139.4$ pg per milliliter in the sotatercept 0.7-mg group, as compared with an increase of $310.4 \pm 151.3$ pg per milliliter in the placebo group. The least-squares mean difference between the sotatercept 0.3 -mg group and the placebo group was -931.5 pg per milliliter (95\% CI, -1353.2 to -509.7), and the least-squares mean difference between the sotatercept $0.7-\mathrm{mg}$ group and the placebo group was -651.0 pg per milliliter $(95 \% \mathrm{CI}$, -1043.3 to -258.7 ) (Figs. S5 and S6 and Table 2).

The mean change in systolic excursion of the tricuspid annular plane did not differ significantly between the sotatercept groups and the placebo group. The WHO functional class improved from baseline by at least one class in 4 patients $(12 \%)$ in the placebo group, in 10 patients (31\%) in the sotatercept $0.3-\mathrm{mg}$ group, and in 7 patients (17\%) in the sotatercept $0.7-\mathrm{mg}$ group. Clinical worsening events occurred in 2 patients $(6 \%)$ in the placebo group, in no patients in the sotatercept $0.3-\mathrm{mg}$ group, and in 1 patient (2\%) in the sotatercept $0.7-\mathrm{mg}$ group (Table 2). On the basis of the scores on the quality-of-life, activity limitation, and symptom scales of the Cambridge Pulmonary Hypertension Outcome Review and the mental and physical component scores on the SF-36, quality of life remained stable for patients in all three groups during the 24-week placebocontrolled treatment period (Table S7).

\section{SAFETY}

The most commonly reported adverse events throughout the trial are listed in Table 3; serious adverse events are detailed in Table S8. Adverse events of CTCAE grade 3 or higher were reported in 3 patients $(9 \%)$ in the sotatercept $0.3-\mathrm{mg}$ group, in 11 patients (26\%) in the sotatercept $0.7-\mathrm{mg}$ group, and in 5 patients (16\%) in the placebo group (Table S9).

Thrombocytopenia was the most common adverse event of special interest, occurring in 2 patients (6\%) in the sotatercept $0.3-\mathrm{mg}$ group and in 5 patients (12\%) in the sotatercept $0.7-\mathrm{mg}$ group but in no patients in the placebo group. No events of thrombocytopenia-related bleeding were reported during the trial, and no patient received a platelet transfusion. Hemoglobin increase was reported as an adverse event in 1 patient (3\%) in the sotatercept $0.3-\mathrm{mg}$ group and in 7 patients (17\%) in the sotatercept 0.7 -mg group but in no patients in the placebo group. Details of the adverse events of thrombocytopenia and hemoglobin increase are provided in the Supplementary Appendix.

In accordance with protocol-specified criteria, 3 patients ( 1 in the sotatercept $0.3-\mathrm{mg}$ group and 2 in the sotatercept $0.7-\mathrm{mg}$ group) were withdrawn from the trial because their hemoglobin levels had risen above $18 \mathrm{~g}$ per deciliter and because they had undergone protocol-mandated phlebotomy. The occurrence of thrombocytopenia resulted in the discontinuation of sotatercept in 1 patient and the withdrawal of consent in another patient; both were in the sotatercept $0.7-\mathrm{mg}$ group. One patient in the sotatercept 0.7-mg group died from cardiac arrest during the trial.

\section{DISCUSSION}

In the PULSAR trial, 24 weeks of treatment with sotatercept resulted in a reduction in pulmonary vascular resistance that was significantly greater than the reduction seen with placebo in patients with pulmonary arterial hypertension. Improvements from baseline in exercise capacity (as assessed by 6-minute walk distance) and NT-proBNP levels were also noted with sotatercept. All the patients had been receiving background therapy with approved agents for pulmonary arterial hypertension before enrollment in the trial and continued the treatment during the trial. The greater degree of reduction in pulmonary vascular resistance as compared with placebo was seen at both dose levels of sotatercept, with the higher dose resulting in a 34\% reduction from baseline. Thrombocytopenia and an increased hemoglobin level were the most common hematologic adverse events. One patient in the higherdose sotatercept group died from cardiac arrest.

Sotatercept was shown to reduce pulmonary vascular resistance in patients receiving background monotherapy, double therapy, or triple therapy, including those who were receiving 
prostacyclin infusion therapy. The decrease in pulmonary vascular resistance in the sotatercept groups was achieved by reducing the mean pulmonary artery pressure, without causing a substantial change in cardiac output or pulmonary artery wedge pressure. Preclinical evidence suggests that sotatercept has a direct effect on pulmonary vascular remodeling, which may explain its clinical effect on pulmonary artery pressure. ${ }^{17}$

Thrombocytopenia was the most common adverse event of special interest but was not associated with bleeding events in this trial; all the events were reversible after delay, reduction, or discontinuation of sotatercept or placebo. The observed increases in hemoglobin levels are consistent with the erythropoietic effects of sotatercept seen in previous clinical trials. ${ }^{18-24}$ Mean hemoglobin levels remained elevated throughout the treatment period in both sotatercept groups, and 3 patients discontinued sotatercept or placebo in accordance with the protocol owing to hemoglobin levels that exceeded the prespecified safety margin of $18 \mathrm{~g}$ per deciliter.

This trial has some limitations. First, as with other phase 2 trials, the sample size was relatively small; however, allowances made for expected dropout rates in each treatment group were sufficient to show improvements with respect to the primary and secondary end points. Second, the duration of the trial was brief at 24 weeks, and the longer-term effects of sotatercept in this clinical context have not been established. Third, the trial was not designed to study the effects of sotatercept on clinical outcomes, including mortality, and this would be important to address in future trials.

Sotatercept is a first-in-class therapeutic fusion protein that targets an imbalance in activingrowth differentiation factor and BMP pathway signaling. In this trial, treatment with sotatercept reduced pulmonary vascular resistance among patients with pulmonary arterial hypertension who were receiving stable background therapy, including prostacyclin infusion therapy. Concordant improvements from baseline in 6-minute walk distance and NT-proBNP levels were also observed. Additional trials, including a phase 3 trial, are ongoing or planned.

Supported by Acceleron Pharma.

Disclosure forms provided by the authors are available with the full text of this article at NEJM.org.

A data sharing statement provided by the authors is available with the full text of this article at NEJM.org.

We thank the patients and families who participated in the PULSAR trial; the investigators and their research teams who collaborated on the trial; current and former personnel at Acceleron Pharma, including Patrick Andre, Carolyn J. Barron, Erica Davis, Shuree Harrison, Ravindra Kumar, Musa Mutyaba, John Oram, John Quisel, Joseph G. Reynolds, and Carlos Sanmarco; and Zoe Noakes and Sophie Briggs of InterComm International, for medical writing assistance (funded by Acceleron Pharma).

\section{APPENDIX}

The authors' affiliations are as follows: the Department of Respiratory and Intensive Care Medicine, Hôpital Bicêtre, Assistance Publique-Hôpitaux de Paris, INSERM Unité Mixte de Recherche 999, Université Paris-Saclay, Le Kremlin-Bicêtre, France (M.H., D.M.); the Division of Cardiovascular Medicine, Department of Internal Medicine, University of Michigan Health System, Ann Arbor (V.M.); the National Heart and Lung Institute, Imperial College London, and the National Pulmonary Hypertension Service, Hammersmith Hospital, Imperial College Healthcare NHS Trust, London (J.S.R.G.); the Department of Medicine, George Washington University, Washington, DC (M.G.-M.); the Department of Respiratory Medicine, Hannover Medical School, and the German Center for Lung Research both in Hannover, Germany (M.M.H., K.M.O.); the Division of Pulmonary, Critical Care and Sleep Medicine, Tufts Medical Center (I.R.P.), and the Division of Pulmonary and Critical Care Medicine, Department of Medicine, Brigham and Women's Hospital (A.W.), Boston, and Acceleron Pharma, Cambridge (S.M., J.B., P.G.L., J.O.P.) - all in Massachusetts; the Pulmonary Division-Heart Institute, University of São Paulo Medical School, São Paulo (R.S.), and Complexo Hospitalar Santa Casa de Porto Alegre, Pulmonary Vascular Research Institute, Porto Alegre (G.M.) - both in Brazil; the Department of Cardiology, Centro de Investigación en Red en Enfermedades Cardiovasculares, Hospital Universitario 12 de Octubre, Universidad Complutense, Madrid (P.E.S.); Arizona Pulmonary Specialists, Phoenix (J.F.); and the Divisions of Pulmonary Sciences and Critical Care Medicine, and Cardiology, University of Colorado, Anschutz Medical Campus, Aurora (D.B.B.).

\section{REFERENCES}

1. Humbert M, Guignabert C, Bonnet S, et al. Pathology and pathobiology of pulmonary hypertension: state of the art and research perspectives. Eur Respir J 2019; 53(1):1801887.

2. Galiè N, Humbert M, Vachiery JL, et al. 2015 ESC/ERS guidelines for the diagnosis and treatment of pulmonary hypertension. Eur Heart J 2016;37:67-119.

3. Humbert M, Lau EM, Montani D, Jaïs
X, Sitbon O, Simonneau G. Advances in therapeutic interventions for patients with pulmonary arterial hypertension. Circulation 2014;130:2189-208.

4. Sitbon O, Gomberg-Maitland $M$, Granton J, et al. Clinical trial design and new therapies for pulmonary arterial hypertension. Eur Respir J 2019;53(1):1801908. 5. Farber HW, Miller DP, Poms AD, et al. Five-year outcomes of patients enrolled in the REVEAL Registry. Chest 2015;148: 1043-54.

6. Kylhammar D, Kjellström B, Hjalmarsson $\mathrm{C}$, et al. A comprehensive risk stratification at early follow-up determines prognosis in pulmonary arterial hypertension. Eur Heart J 2018;39:4175-81.

7. Olsson KM, Richter MJ, Kamp JC, et al. Intravenous treprostinil as an add-on therapy in patients with pulmonary arterial 
hypertension. J Heart Lung Transplant 2019;38:748-56.

8. Deng Z, Morse JH, Slager SL, et al. Familial primary pulmonary hypertension (gene PPH1) is caused by mutations in the bone morphogenetic protein receptor-II gene. Am J Hum Genet 2000;67:737-44.

9. Atkinson C, Stewart S, Upton PD, et al. Primary pulmonary hypertension is associated with reduced pulmonary vascular expression of type II bone morphogenetic protein receptor. Circulation 2002;105: $1672-8$.

10. Morrell NW, Yang X, Upton PD, et al. Altered growth responses of pulmonary artery smooth muscle cells from patients with primary pulmonary hypertension to transforming growth factor-beta(1) and bone morphogenetic proteins. Circulation 2001;104:790-5.

11. Guignabert C, Humbert M. Targeting transforming growth factor- $\beta$ receptors in pulmonary hypertension. Eur Respir J 2021;57(2):2002341.

12. Yang X, Long L, Southwood $M$, et al. Dysfunctional Smad signaling contributes to abnormal smooth muscle cell proliferation in familial pulmonary arterial hypertension. Circ Res 2005;96:1053-63. 13. Yndestad A, Larsen K-O, Oie E, et al. Elevated levels of activin A in clinical and experimental pulmonary hypertension. J Appl Physiol (1985) 2009;106:1356-64. 14. Morrell NW, Aldred MA, Chung WK, et al. Genetics and genomics of pulmo- nary arterial hypertension. Eur Respir J 2019;53(1):1801899.

15. David L, Mallet C, Mazerbourg S, Feige J-J, Bailly S. Identification of BMP9 and BMP10 as functional activators of the orphan activin receptor-like kinase 1 (ALK1) in endothelial cells. Blood 2007; 109:1953-61.

16. Upton PD, Davies RJ, Trembath RC, Morrell NW. Bone morphogenetic protein (BMP) and activin type II receptors balance BMP9 signals mediated by activin receptor-like kinase-1 in human pulmonary artery endothelial cells. J Biol Chem 2009;284:15794-804.

17. Yung LM, Yang P, Joshi S, et al. ACTRIIAFc rebalances activin/GDF versus BMP signaling in pulmonary hypertension. Sci Transl Med 2020;12(543):eaaz5660.

18. Raftopoulos H, Laadem A, Hesketh PJ, et al. Sotatercept (ACE-011) for the treatment of chemotherapy-induced anemia in patients with metastatic breast cancer or advanced or metastatic solid tumors treated with platinum-based chemotherapeutic regimens: results from two phase 2 studies. Support Care Cancer 2016; 24:1517-25.

19. Abdulkadyrov KM, Salogub GN, Khuazheva NK, et al. Sotatercept in patients with osteolytic lesions of multiple myeloma. Br J Haematol 2014;165:814-23. 20. Ruckle J, Jacobs M, Kramer W, et al. Single-dose, randomized, double-blind, placebo-controlled study of ACE-011
(ActRIIA-IgG1) in postmenopausal women. J Bone Miner Res 2009;24:744-52.

21. Komrokji R, Garcia-Manero G, Ades $\mathrm{L}$, et al. Sotatercept with long-term extension for the treatment of anaemia in patients with lower-risk myelodysplastic syndromes: a phase 2, dose-ranging trial. Lancet Haematol 2018;5(2):e63-e72.

22. Cappellini MD, Porter J, Origa R, et al. Sotatercept, a novel transforming growth factor $\beta$ ligand trap, improves anemia in $\beta$-thalassemia: a phase II, open-label, dose-finding study. Haematologica 2019; 104:477-84.

23. Coyne DW, Singh HN, Smith WT, et al. Sotatercept safety and effects on hemoglobin, bone, and vascular calcification. Kidney Int Rep 2019;4:1585-97.

24. Sherman ML, Borgstein NG, Mook L, et al. Multiple-dose, safety, pharmacokinetic, and pharmacodynamic study of sotatercept (ActRIIA-IgG1), a novel erythropoietic agent, in healthy postmenopausal women. J Clin Pharmacol 2013;53:1121-30. 25. Declaration of Helsinki (1964). BMJ 1996;313:1448-9.

26. van Delden JJ, van der Graaf R. Revised CIOMS international ethical guidelines for health-related research involving humans. JAMA 2017;317:135-6.

27. International Council for Harmonisation. Guidelines for good clinical practice. London: European Medicines Agency, 2002. 